\title{
La naissance de l’opéra. Traduire la pensée musicale en devenir
}

Adrien Alix, Françoise Decroisette et Joël Heuillon

\section{(2) OpenEdition}

\section{Édition électronique}

URL : http://journals.openedition.org/traduire/1546

DOI : 10.4000/traduire.1546

ISSN : 2272-9992

\section{Éditeur}

Société française des traducteurs

\section{Édition imprimée}

Date de publication : 1 décembre 2018

Pagination : 53-62

ISSN : 0395-773X

\section{Référence électronique}

Adrien Alix, Françoise Decroisette et Joël Heuillon, «La naissance de l'opéra. Traduire la pensée

musicale en devenir », Traduire [En ligne], 239 | 2018, mis en ligne le 01 décembre 2018, consulté le 29 juin 2019. URL : http://journals.openedition.org/traduire/1546 ; DOI : 10.4000/traduire.1546 


\title{
La naissance de l'opéra. Traduire la pensée musicale en devenir
}

\author{
Adrien Alix, Françoise Decroisette, Joël Heuillon
}

\section{Introduction}

La musique est un art de médiation : l'œuvre ne paraît que lorsqu'un interprète la rend perceptible. Une partition est comme un canevas de commedia dell'arte; plus ou moins nourrie d'indications selon les époques, elle est particulièrement lacunaire durant la période baroque (1600-1750). L'interprète, qui se fait alors herméneute, doit opérer des choix quant à la réalisation sonore des signes inscrits. Traduire l'art, c'est, pour lui, passer de ce système muet à une proposition sonore éloquente, qui parle à tous. Notre volonté est de l'aider dans cette tâche : traduire l'art signifie, pour nous, mettre à disposition en français, en les élucidant, les métatextes qui entourent les œuvres, de sorte qu'ils permettent à l'interprète de prendre des décisions, non pas selon sa seule fantaisie, mais dans un cadre balisé par des savoirs.

Après quelques expériences érudites au xixe siècle et au début du Xxe siècle, la musique baroque s'est imposée peu à peu dans les pratiques musicales à partir des années 1950, avec une accélération dans les années 1980 et 1990, se positionnant alors en tête des concerts et des ventes de disques de musique "classique".

Force est de constater que la curiosité des pionniers du revival de la musique ancienne (Harnoncourt, Deller, Malgoire) a laissé lentement mais sûrement place à une académisation de la pratique de ces répertoires, singulièrement pour la musique dite "baroque ". Ainsi la musique ancienne s'est-elle constituée progressivement, via son enseignement dans les conservatoires, en diverses traditions d'interprétation plus soucieuses de répondre aux attentes d'un marché florissant que de restituer à ces musiques leur efficacité première, à défaut d'une vérité historique souvent illusoire.

On pourrait avancer que cette musique se passe fort bien des savoirs universitaires, que sa puissance d'expression comble aisément les quelques siècles qui nous en séparent ; certes, la mélodie, l'harmonie et la beauté des timbres se passent d'explications... Mais c'est une forme de trahison que de réduire ces musiques à leur sensualité. En effet, la volonté de faire 
sens, de dire en musique, est fondatrice des innovations musicales baroques dans des genres et des formes où musique et poésie sont consubstantielles, triomphant avec la naissance de l'opéra (favola in musica). II faut donc admettre la nécessité d'une réflexion préalable, d'une intercession entre l'œuvre et l'interprète qui lui permette de se réapproprier les outils conceptuels du temps pour comprendre la pensée qui sous-tend l'œuvre. Difficile alors d'échapper à l'étude des textes, seules sources à même de nous renseigner. Ainsi la recherche universitaire ou extra-universitaire devient-elle l'alliée des interprètes en leur offrant tous les outils utiles aux nécessaires décisions.

C'est dans cet esprit que nous avons constitué l'équipe de recherche Euridice 1600-2000 et entrepris de traduire et publier un certain nombre de textes-clés, fondateurs des grandes évolutions langagières, formelles et esthétiques du premier baroque (env. 1580-1650). Une approche pluridisciplinaire, unissant musicologie, littérature, théâtrologie et études italiennes, nous a semblé judicieuse dans la mesure où l'opéra est conçu dès l'origine comme un art syncrétique qui convoque, au-delà de la poésie et de la musique, les arts visuels et le théâtre. À titre d'illustration de notre démarche, nous reviendrons donc ici sur les difficultés de traduction posées par un certain nombre de concepts cardinaux de cette musique du premier baroque.

\section{La favola in musica}

C'est en 1600 à la cour de Florence que naît l'opéra, dénommé alors favola in musica. Giulio Caccini (1551-1618) et Jacopo Peri (1561-1633) composent l'un et l'autre une Euridice sur un même livret d'Ottavio Rinuccini (1562-1621). Ces deux publications sont accompagnées de préfaces dans lesquelles les deux musiciens rivaux légitiment la nouveauté de leur ouvrage par un discours théorique qui prend source dans les savants entretiens de l'académie informelle animée par le comte Giovanni de' Bardi. Là se réunissaient " les plus éminents musiciens, les grands esprits, les poètes et philosophes "(1) pour réfléchir, parallèlement aux travaux des académies de l'Italie septentrionale, à l'interprétation de la Poétique d'Aristote, notamment pour ce qui concerne la tragédie dont on constate qu'elle ne produit plus alors les effets attestés du spectacle antique et dont les auteurs et le public se détournent. L'hypothèse que la tragédie antique ait été chantée de part en part, prônée par la Camerata de' Bardi, rend nécessaire l'élaboration d'une musique nouvelle capable de seconder efficacement l'action dramatique. Cela aboutira à l'opéra, ainsi qu'à des musiques vocales intimistes comme le madrigal et la cantate, également publiées avec des préfaces théoriques.

(1) Caccini (1993), p. 86. 
Traduire ce type de textes amène à relever des défis de restitution du sens dont nous donnerons quelques exemples. La séparation des champs disciplinaires et la dévaluation lexicale dotent les mots de sens différents ; l'opéra oblige à passer outre le cloisonnement artificiel des disciplines et à restituer leur épaisseur sémantique à des termes dont l'ambiguïté marque davantage la complexité que l'indétermination.

La locution favola in musica, par laquelle les créateurs de l'opéra désignent les premiers textes poétiques destinés à être mis en musique, dérive d'Aristote. Celui-ci définit dans la Poétique les parties constitutives de la tragédie et donne la première place à "l'assemblage des actions accomplies " qui constituent l'histoire représentée, ou l'intrigue(2). Pour nommer cet assemblage d'actions, mais aussi leur représentation, Aristote emploie le terme mythos qui, dans les traductions latines ultérieures, devient fabula, puis, en italien, favola. Dans les tragédies, la favola investit l'ensemble des opérations de la rhétorique : elle a trait aux mœurs ou caractères, à l'invention, à l'élocution, à la mélopée et en dernier lieu à l'appareil scénique. II faut rapprocher ce terme de la favola pastorale, la tragicomédie pastorale, genre dramatique mixte souvent entremêlé de chants qui, dans le dernier tiers du xvie siècle, s'affirme face à la tragédie, comme le déclare la prosopopée de la Tragédie dans le prologue de L'Euridice de Rinuccini. Les premières favole in musica puisent leurs histoires dans les mythes antiques dont le caractère allégorique convient aux noces princières ; mythes musicaux (Orphée) et métamorphoses (Daphné, Céphale et Procris) permettent un large déploiement du merveilleux scénique. On ne saurait donc confondre la favola avec la fable en tant que forme littéraire brève telle que la pratiquent Ésope, Phèdre ou plus tard La Fontaine, et telle que nous l'entendons aujourd'hui.

\section{Le recitar cantando et la basse continue}

L'expression recitar cantando apparaît explicitement à la fin de la longue préface à la Rappresentazione di Anima e di Corpo d'Emilio de' Cavalieri. Ce syntagme vise moins à désigner le nouveau style de chant qu'à encadrer les interprètes en vue de la représentation de l'œuvre. Recitare est ici utilisé clairement dans le sens de rappresentare (" représenter " ou " jouer ") : les deux termes, interchangeables dans les sources contemporaines, s'inscrivent dans le champ sémantique de la mimesis aristotélicienne et impliquent un dispositif spectaculaire plaçant l'acteur face à un public. Cela suffit à distinguer ces nouvelles musiques de la polyphonie du siècle précédent dans laquelle l'incarnation d'un personnage par un comédien-chanteur est problématique et où l'enjeu n'est souvent pas tant de représenter pour un public que de faire corps par la musique(3).

(2) Aristote, Poétique, VI-VII, 1450a-1450b (p. 38-40 dans l'édition référencée en bibliographie).

(3) À l'inverse, la représentation s'apparente à une confrontation, à une joute, rituel sacré et spectaculaire. Ainsi le verbe recitare peut-il traduire l'agonizesthai des Grecs. Voir Siekiera, entrée gareggiare, p. 140-141. 
Le recitar cantando prend la forme d'une musique à voix seule avec accompagnement de la basse continue, ce que le musicographe Giovanni Battista Doni désigne ultérieurement par monodia(4). La profération par une voix seule permet d'une part une plus grande intelligibilité du texte, et d'autre part de penser le chanteur comme un personnage doté d'une identité singulière et stable.

Autour de recitar cantando se forgent d'autres expressions apparentées qui ne renvoient pas nécessairement à la favola in musica. En effet, l'écriture spécifique à ces premiers opéras se retrouve bientôt dans un répertoire vocal soliste da camera, c'est-à-dire " de chambre ", par opposition à la musique da teatro(5), d'où un certain nombre d'expressions comme stile recitativo, stile ou genere rappresentativo(6). Le stile recitativo désigne ce mode déclamatoire chanté du texte mis en musique, selon les impératifs de la prosodie, de la métrique et du sens ; il suppose que chaque texte induise une proposition musicale originale, la répétition étant exclue. L'interprète garantit, par son incarnation, la directivité de la proposition musicale avec plus ou moins d'intensité expressive, déployant à propos toutes les ressources de son art (dynamiques, articulations, ornementation, couleurs vocales, etc.).

Le parlar cantando (" parler en chantant "), équivalent à in armonia favellare (" discourir en musique "), insiste sur la présence d'un discours qui produit du sens par le moyen du chant, et s'applique au stile recitativo. Monteverdi distinguera ensuite le cantar parlando, qui signifie " chanter en parlant " et désigne les musiques ariose où une seule et même mélodie est utilisée pour énoncer le texte de plusieurs strophes ou couplets.

Quant à la basse continue, traduction littérale de basso continuo, elle désigne avant tout une forme d'écriture synthétique de l'accompagnement instrumental qui se présente de la manière suivante :

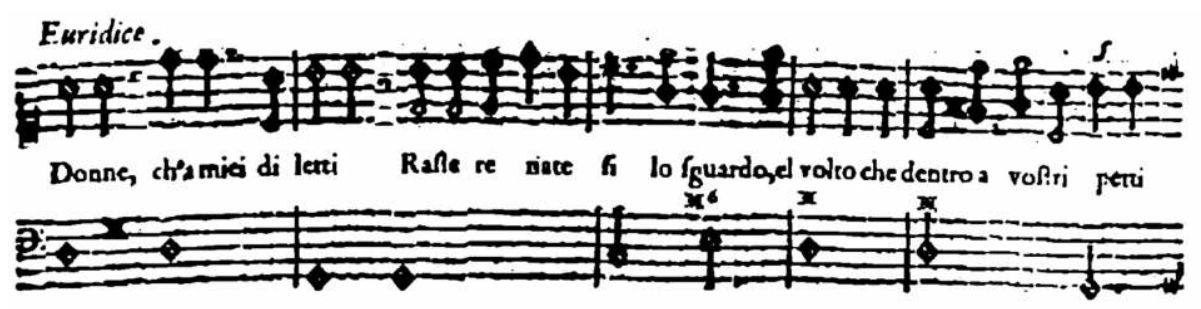

Peri, Le musiche sopra l'Euridice, p. 5

(4) Cf. Baron. La musicologie moderne emploie généralement " monodie " pour l'opposer à " polyphonie " dans un usage beaucoup plus large que celui que lui réservait Doni.

(5) La terminologie varie alors d'une publication à l'autre : madrigali, arie, cantade, nuove musiche, etc.

(6) Caccini, dans Le Nuove musiche (1602), parle des œuvres composées « dans ledit style, que j'adoptai ensuite pour les favole qui furent représentées en chantant [rappresentate cantando] à Florence ". Caccini (1993), p. 89 [traduction légèrement révisée, à paraître bientôt sur le site Euridice 1600-2000]. 
Les instrumentistes accompagnateurs déduisent des quelques notes écrites sur la portée inférieure, surmontées de rares signes et chiffres, un environnement harmonique complet résultant de l'assimilation de deux sources distinctes et complémentaires : d'une part, un ensemble de règles héritées de l'art du contrepoint savant et, d'autre part, des pratiques d'accompagnement improvisé ancrées dans l'esprit et le geste. Les termes basso continuo, continuato ou seguente renvoient par ailleurs à la pratique qui découle de cette notation et dont la théorisation est postérieure(7).

La notation de la basse continue apparaît simultanément à l'invention de la monodie et de l'opéra. Elle semble en effet particulièrement adaptée à la liberté agogique du recitar cantando, et contribue avec le chant, par l'introduction de figures qui réalisent rhétoriquement l'harmonie, à révéler efficacement le sens et l'énergie du texte, car "les consonances et toute l'harmonie sont sujettes et soumises aux paroles, et non le contraire "(8). L'usage de la basse continue s'étend cependant bientôt à tous les genres musicaux, sacrés comme profanes.

\section{Grâce et sprezzatura}

Dans les préfaces à L'Euridice puis aux Nuove musiche, Giulio Caccini introduit la sprezzatura dans le champ musical afin de "s'approcher au plus près du parler naturel „(9). La negligentia diligens de Cicéron anticipe partiellement le concept de sprezzatura, terme difficile à définir et à traduire(10), forgé en 1528 par Baldassare Castiglione dans Le livre du courtisan à partir du verbe sprezzare (dédaigner, mépriser), qui caractérise la noblesse propre à l'homme de cour. Castiglione préconise en effet pour le courtisan "d'utiliser en chaque chose une certaine sprezzatura, qui cache l'artifice et montre que ce que l'on fait et dit est fait sans effort, et presque sans y penser "(11). II s'agit donc de cacher l'art (au sens de tékhnê, technique) par l'art même et de faire montre de facilité et de rapidité, produisant un effet de naturel et d'évidence.

La sprezzatura devient sous la plume de Caccini l'élément central du code performatif du musicien interprète. La sprezzatura désigne donc une posture générale destinée à dissimuler l'effort pour se consacrer pleinement à l'expression du texte mis en musique(12). La publication

(7) Cf. Williams et Ledbetter.

(8) Agazzari, p. 5., "le consonanze e tutta l'armonia sono soggette e sottoposte alle parole, e non per il contrario ".

(9) Voir notre traduction de la préface à L'Euridice présentée sur le site Euridice 1600-2000. Voir aussi Caccini (1993), p. 88 et 120.

(10) Les traductions françaises hésitent depuis le xvie siècle entre "nonchalance ", " un certain mépris et nonchalance ", et plus récemment " désinvolture " (Castiglione (2009), p. 54). L'expression " négligence diligente " traduit quant à elle la source cicéronienne. Voir Fumaroli, p. 54, note 32.

(11) Castiglione (2017), I, ch. 26.

(12) Marin Mersenne, musicographe français de cette période, est frappé par la force expressive des chanteurs qu'il observe en Italie et qui semblent atteints des passions mêmes qu'ils représentent. 
de l'effort ("l'affectation ", selon Caccini qui se réfère de nouveau à Castiglione(13)) est alors la chose à fuir, car elle annule la convention de représentation, ramenant le chanteur à la seule difficulté technique de l'exécution et ruinant ainsi toute l'entreprise artistique. Seule la sprezzatura alliée au giudizio (le jugement acéré) garantit la réalisation efficace du programme sémantique et pathétique porté par le texte fusionné à la musique.

Dans le même chapitre, Castiglione approfondit la définition de la sprezzatura dans son rapport à la grazia, que l'on peut traduire par " grâce ". Si ces deux notions visent à produire un même effet, la grâce est innée, tandis que la sprezzatura ne l'est pas forcément ; elle peut être mise en œuvre pour masquer l'effort ou l'intention qui animent le courtisan dans ses gestes et sa conversation. Mais au bout du compte, les deux qualités sont indissolublement liées, tout comme elles le sont chez le chanteur éloquent tel que le conçoit Caccini.

Les premières favole in musica étaient destinées à des fêtes princières, et donc aux publics courtisans auxquels elles devaient renvoyer une image gratifiante. Aussi les créateurs florentins et romains sont-ils allés puiser à nouveau chez Castiglione la grazia, présentée comme la qualité essentielle de l'homme de cour, une disposition naturelle, innée, presque indéfinissable. L'utilisation par les compositeurs des premières favole in musica du terme grazia, renvoie sans doute aussi aux conceptions de Marsile Ficin sur les pouvoirs thaumaturgiques de la musique et de la danse, lecture qui inspire les membres de la Camerata de' Bardi(14).

Par la suite, les grazie (au pluriel) désigneront également une certaine catégorie de figures ornementales du chant dont l'exécution judicieuse garantit un effet de grâce. Il est intéressant de noter ici la confusion entre l'effet (la grazia) et la cause (les grazie)(15).

\section{Des différents usages de la voix}

L'accumulation de termes polysémiques et le recours à des concepts mal définis rend souvent l'explication de texte et donc la traduction complexes. Un passage de la préface de Peri requiert à ce titre une attention toute particulière :

(13) Castiglione recommande en effet de " fuir tant que possible, comme un écueil très âpre et périlleux, l'affectation " ( fuggir quanto più si po, e come un asperissimo e pericoloso scoglio, la affettazione ").

(14) Cf. Ficin.

(15) On retrouve d'ailleurs une ambiguïté semblable dans le terme espagnol ayre, qui semble signifier dans les traités du xvIe siècle le " bon goût " ou la " grâce ", mais qui acquiert au début du siècle suivant une signification plus technique, car il en vient à désigner l'inégalité des valeurs brèves (comparable à la fameuse "inégalité à la française " qui ne serait donc pas si française que ça, et que Caccini décrit déjà comme un raffinement d'exécution parmi les figures de la sprezzatura). Voir Noguera Guillén. 
[...] je considérai que cette sorte de voix que les Anciens assignèrent au chant et qu'ils appelaient Diastématique (comme retenue et suspendue) pouvait quelque peu se hâter et prendre un cours tempéré entre les mouvements lents et suspendus du chant, et ceux précipités et véloces de la parole, et s'accommoder à ce que je me proposais [...] en se rapprochant de l'autre [sorte de voix], celle du discours, qu'ils appelaient continue.(16)

II tente ici de situer le recitar cantando entre la voix lyrique et la voix quotidienne, celle du "parler ordinaire ". Cette tentative théorique repose sur une lecture attentive des sources antiques dans la continuité des débats de la Camerata. Peri développe et actualise le chapitre 12 du premier livre du Traité de la musique de Boèce, compilation du savoir musical spéculatif antique rédigée au début du vie siècle de notre ère. Boèce oppose en effet la voix continue et la voix diastématique, et ajoute une troisième voix intermédiaire, particulièrement adaptée à la récitation de l'épopée.

Cette opposition met en jeu deux paramètres : d'abord, la voix diastématique se fixe sur des hauteurs différenciées, elle s'organise selon une échelle de sons qui forment une suite intervallique de valeurs discrètes (la gamme des modernes ou l'hexacorde des anciens). Le mot grec diastema signifie d'ailleurs « intervalle ", différence de hauteur entre deux sons. À l'inverse, la voix continue parcourt une infinité de hauteurs sans jamais se stabiliser sur un degré précis : ce sont les intonations du parler ordinaire. II est par ailleurs question de vitesse : la voix diastématique, chantée, a un débit nettement inférieur à celui du parler.

Cherchant une voie médiane entre ces deux opposés (de même que la tragicomédie pastorale constitue un genre mixte), Peri propose donc un chant discursif, un favellare in armonia au débit moyen, ni trop distendu, ni trop précipité. La difficulté est supérieure en ce qui concerne les hauteurs : comment la voix peut-elle procéder à la fois par intervalles et de manière continue? Peri a alors recours au génie de la langue, abandonnant la veste du philologue pour celle du créateur :

Je m'aperçus de même que quelques mots dans notre langue parlée peuvent porter une harmonie, et qu'on passe ensuite au fil de la parole par beaucoup d'autres qui ne le peuvent pas, jusqu'à ce qu'on en retrouve un autre capable de susciter une nouvelle consonance.

La sélection de ces syllabes obéit à un double impératif linguistique et poétique : elle s'appuie certes sur les accents toniques de l'italien (accents d'intensité et de durée évidents dans les intonations de la langue parlée), mais aussi sur l'imitation de l'expression des passions.

[...] ayant considéré les tons et les accents qui nous servent dans la douleur, dans la joie et autres choses semblables, je fis se mouvoir la basse à leur rythme, tantôt plus, tantôt moins, en fonction des passions, et je la tins ferme parmi les intervalles consonants et dissonants, [...].

(16) Extrait de la traduction présentée sur le site Euridice 1600-2000. 
Le langage théorique de Peri est complexe, heurté, saturé de références. La nouveauté s'exprime avec difficulté, car elle ne fait pas table rase du passé mais s'inscrit au contraire dans la continuité de débats riches nourris de lectures anciennes. Mais la proposition musicale qui en découle s'exprime au contraire avec fluidité et bonheur lorsque l'on prend la peine de déchiffrer ce langage !

\section{Conclusion}

Ces quelques exemples montrent assez, sans pour autant l'épuiser, la polysémie des concepts convoqués dans ces textes fondateurs des innovations musicales du premier baroque, et laissent entrevoir l'ampleur du travail herméneutique auquel doit se livrer l'interprète responsable qui veut redonner une parole efficace à ces musiques, raviver leur substance première, déployer puissamment pour le spectateur l'énergie qu'elles recèlent.

Plutôt que de traduire par un terme français qui ne saurait faire entendre l'épaisseur sémantique (historique, esthétique, poétique, technique) des termes-concepts d'origine, nous préférons la plupart du temps conserver dans le texte français le mot italien, à l'instar de sprezzatura, quitte à l'accompagner d'un apparat critique conséquent. Notre programme éditorial prévoit donc d'établir un lexique transversal conçu comme une base de données évolutive qui se nourrirait des occurrences supplémentaires de ces termes dans les textes nouvellement intégrés au corpus. Cela est rendu possible par notre choix d'édition en ligne, qui permet l'ajout, le remords, l'amendement, la correction.

Si l'interprète constitue le public privilégié de cette entreprise, elle s'inscrit naturellement dans le débat académique de la musicologie francophone, mais s'adresse plus largement aux chercheurs des disciplines connexes désireux de se confronter à ces textes dans une démarche comparatiste. Ainsi certains des concepts identifiés dans ces pages trouvent-ils une résonance certaine dans la recherche sur le maniérisme et le baroque.

Dans un souci de vulgarisation, et pour nourrir les esprits curieux des temps modernes, cette somme de savoirs sera disponible pour qui s'interroge sur les fondements esthétiques d'un baroque qui renferme sous une apparence séduisante une profondeur qui en légitime la présence dans le paysage artistique contemporain. 
L'équipe de recherche Euridice 1600-2000 se forme à l'occasion du quatrième centenaire de la création de Le musiche sopra l'Euridice de Peri/Rinuccini à Florence en 1600. Associant chercheurs et interprètes, elle se propose d'étudier les relations entre musique et poésie (innovations musicales langagières et formelles) à l'aube de l'âge baroque, dans les cours du Nord de l'Italie (Florence, Mantoue, Ferrare). Elle se distingue par une approche interdisciplinaire et expérimentale. Ainsi, en novembre 2017, un colloque international autour d'Orphée se double-t-il d'une représentation de L'Orfeo de Monteverdi à I'Université Paris 8.

Françoise Decroisette est professeure émérite des universités en études italiennes et histoire des spectacles en Italie et en France. Elle poursuit une intense activité de traduction.

Joël Heuillon est maître de conférences en musicologie à l'université Paris 8. II travaille sur les musiques baroques passées au crible de la rhétorique et dirige l'équipe Euridice 1600-2000.

Adrien Alix est musicien et doctorant en littératures comparées et musicologie à l'université de Corse et de Paris 8. II s'intéresse à la présence de la musique dans l'œuvre du poète Giambattista Marino et à son influence sur la poésie en musique du premier baroque.

\section{Bibliographie}

AGAZZARI Agostino, 1607, Del sonare sopra'l basso con tutti li stromenti e dell'uso loro nel conserto, Siena (Italie), Falcini.

ARISTOTE, 1969, Poétique, Paris, Les Belles Lettres [édition et traduction de Janet Hardy].

BARON John H., 1968, "Monody: A Study in Terminology " in The Musical Quarterly, 54(4), p. $462-474$.

BOĖCE [Boethius Anicius Manlius Severinus], 2004, Traité de la musique, Turnhout (Belgique), Brepols [présentation et traduction de Christian Meyer].

CACCINI Giulio, 1600, L'Euridice composta in musica in stile rappresentativo, Firenze (Italie), Marescotti.

CACCINI Giulio, 1602 [1601 dans le calendrier florentin], Le nuove musiche, Firenze (Italie), Marescotti.

CACCINI Giulio, 1993, Préfaces aux Nuove Musiche parues à Florence en 1602 et 1614, Paris, Cahiers GKC, coll. "La musique éloquente " [présentation et traduction de Joël Heuillon].

CASTIGLIONE Baldassare, 2009 [1987], Le livre du courtisan, Paris, Éditions Gérard Lebovici [présentation et traduction d'Alain Pons d'après la version de Gabriel Chappuis (1580)].

CASTIGLIONE Baldassare, 2017, II libro del Cortegiano, Torino (Italie), Einaudi [édition de Walter Barberis]. 
CAVALIERI Emilio De', 1600, Rappresentatione di anima e di corpo, Roma, Muti.

FICIN Marsile, 2007, Théologie platonicienne de l'immortalité des âmes. Livres I-XVIII, Paris, Les Belles Lettres [édition et traduction de Raymond Marcel].

FUMAROLI Marc, 2009 [1980], L'âge de l'éloquence, Genève (Suisse), Droz.

NOGUERA GUILLÉN Jesús, à paraître fin 2018, «L'interprétation de la musique espagnole pour clavier au Siècle d'Or : de la lexicologie à la pratique ", in Diego Pacheco Cristina et García Pérez Amaya (éds.), Le Lexique Musical de la Renaissance, Paris, Garnier.

PERI lacopo, 1601 [1600 dans le calendrier florentin], Le musiche sopra l'Euridice del Sig. Ottavio Rinuccini, Firenze (Italie), Marescotti.

RINUCCINI Ottavio, 1600, L'Euridice, Firenze (Italie), Giunti.

SIEKIERA Anna, 2000, Tradurre per musica. Lessico musicale e teatrale nel cinquecento, Paris, Les Belles Lettres, coll. "Cahiers d'Accademia ".

WILLIAMS Peter et LEDBETTER David, 2001, "Continuo » in Grove Music Online, http:////www.oxfordmusiconline.com/grovemusic/view/10.1093/gmo/9781561592630.001.0001/ omo-9781561592630-e-0000006353, consulté le 05/10/2018.

\section{Webographie}

EURIDICE 1600-2000, 2018, Éditions en ligne, France, Université Paris 8, http://www.musique.univ-paris8.fr/orphee/spip.php?rubrique360, consulté le 05/10/2018. 